\title{
HERBORIZAÇÃO DE ORCHIDACEAE COM PSEUDOBULBOS ESPESSADOS
}

\author{
Adarilda Petini-Benelli*
}

\begin{abstract}
RESUMO: Grande parte dos gêneros de Orchidaceae apresenta estruturas caulinares engrossadas denominadas pseudobulbos, os quais contêm grande quantidade de carboidratos e água, formando uma mucilagem densa e volumosa. A presente técnica propõe a remoção dessa mucilagem facilitando a herborização e acelerando a secagem da amostra. Como resultado, obtém-se uma exsicata com ótimo aspecto e pouco volume, preservando em alguns casos parte de sua coloração natural.
\end{abstract}

PALAVRAS-CHAVE: Catasetinae; Exsicata; Laeliinae; Mucilagem; Oncidiinae.

\section{HERBORIZATION OF ORCHIDACEAE WITH THICKENED PSEUDOBULBS}

\begin{abstract}
Most Orchidaceae genera have thickened caulinary structures called pseudobulbs containing great amounts of carbohydrates and water, forming thick and volumetric mucilage. Current technique suggests the removal of the mucilage to improve herborization and accelerate the drying of the sample. Exsiccates are obtained with a good aspect and small volume, conservating their natural hue.
\end{abstract}

KEY WORDS: Exsicates; Mucilage; Catasetinae; Laeliinae; Oncidiinae.

\section{INTRODUÇÃO}

Muitas espécies de Orchidaceae têm órgãos de reserva, denominados pseudobulbos, os quais são segmentos caulinares dilatados, geralmente verticais (DRESSLER, 1990; NG; HEW, 2000) comuns em orquídeas epífitas, onde possuem a função de reserva, a qual nas orquídeas terrestres é assumida pelas raízes carnosas/

\footnotetext{
Doutoranda do Programa de Pós-graduação em Ecologia e Conservação da Biodiversidade da Universidade Federal de Mato Grosso (UFMT), Pesquisadora associada ao Herbário UFMT e ao Herbário da Amazônia Meridional (HERBAM), Brasil; E-mail: ada.benelli@gmail.com
} 
tuberizadas (PEDROSO-DE-MORAES et al., 2012). Compreendendo as espécies de hábito predominantemente epifítico, Epidendroideae é a maior subfamília de Orchidaceae e uma das mais representativas na Região Neotropical (DRESSLER, 1990) e suas subtribos têm muitos registros com coletas de material testemunho.

Em Catasetinae os pseudobulbos podem ser homoblásticos ou heteroblásticos, achatados, ovoides ou fusiformes (HOEHNE, 1942; DRESSLER, 1990; ROMERO, 2009), com grandes reservas de mucilagem armazenada no tecido parenquimático (OLIVEIRA; SAJO, 2001). Laeliinae é a terceira maior subtribo na família (VAN DEN BERG et al., 2009). Seus pseudobulbos são homoblásticos (DRESSLER, 1990), engrossados e espessos, revestidos por epiderme unisseriada e cuticularizada (OLIVEIRA; SAJO, 2001). A região cortical é formada por várias camadas de células parenquimáticas e o cilindro central possui feixes vasculares colaterais com várias camadas de células esclerificadas (OLIVEIRA; SAJO, 2001), o que dificulta o processo de secagem das amostras. As espécies na subtribo Oncidiinae possuem pseudobulbos heteroblásticos e, em diversos gêneros, também se encontram espécies com pseudobulbos bem espessados (DRESSLER, 1990). Similar à Laeliinae, os pseudobulbos de Oncidiinae têm várias camadas de células parenquimáticas na região cortical e de células esclerificadas nos feixes vasculares do cilindro central, o que torna lenta a secagem do conteúdo mucilaginoso neles armazenado (STERN; CARLSWARD, 2007). Em Pleurothallidiinae, a predominância é de ramicaules ou de minúsculos pseudobulbos heteroblásticos, o que facilita o processo de herborização de amostras de suas espécies.

Utilizando Catasetum fimbriatum (C. Morren) Lindl. em seu estudo, Oliveira e Sajo (2001) observaram que, a despeito das variações morfológicas dos pseudobulbos de Orchidaceae, a estrutura anatômica é bastante similar, sendo revestidos por epiderme unisseriada desprovida de tricomas/estômatos e recoberta por cutícula espessada. Internamente são formados por tecido parenquimático onde não é possível estabelecer um limite entre o córtex e o cilindro vascular. Em todos os pseudobulbos estudados, os autores encontraram células pequenas (comumente reservando amido), e células grandes (armazenando água - elementos traqueoidais). Estas últimas são globosas, hialinas e suas paredes apresentavam espessamento secundário. 
Existem descrições de metodologias para coleta e preparação de amostras para herborização (FIDALGO; BONONI, 1989; ROTTA et al., 2008), com grande aplicação em práticas de campo e preparação de exsicatas. No entanto, seguindo as metodologias atualmente difundidas, o coletor encontra dificuldades quando se trata de herborizar Orchidaceae, principalmente espécies que possuam pseudobulbos dilatados. Essas estruturas possuem grande quantidade de material mucilaginoso e água (NG; HEW, 2000; STERN; JUDD, 2001), dificultando o processo de secagem da amostra, o que propicia o desenvolvimento de fungos, comprometendo a amostra ou, mesmo, provocando o seu descarte. Por isso, o desenvolvimento de métodos alternativos para obter um material de secagem mais rápida, sem danificar a amostra mostra-se necessário.

Com o objetivo de que o processo aqui descrito sirva de instrumento auxiliar aos demais pesquisadores que necessitem preparar exsicatas de amostras com estruturas constituídas de tecidos de reserva como, por exemplo, os cladódios de Cactaceae e os pseudobulbos engrossados de Orchidaceae, é que sua divulgação é apresentada, pois o mesmo tem se mostrado muito eficaz no processo de preparação de exsicatas de qualquer amostra que possua estruturas semelhantes.

\section{MATERIAL E MÉTODOS}

As amostras utilizadas na ilustração desse processo são de espécies de Catasetum, principalmente $C$. arietinum F.E.L. Miranda \& K.G. Lacerda cujas fotos compõem a Figura 1.

Considerando que as folhas e flores de Catasetum são membranáceas (HOEHNE, 1942), compostas por tecidos mais delicados do que os que compõem os pseudobulbos, secando mais rapidamente (inclusive as flores femininas, geralmente mais carnosas), estas chegam ao ponto de enegrecerem com a demora na secagem (adquirindo aspecto carbonizado), ficando ressecadas e quebradiças. Para evitar esse resultado e obter uma exsicata com aparência natural, sem danos por queimadura, foi adotado um método que visa otimizar o processo e que consiste na retirada do máximo possível da mucilagem contida no interior do pseudobulbo. 

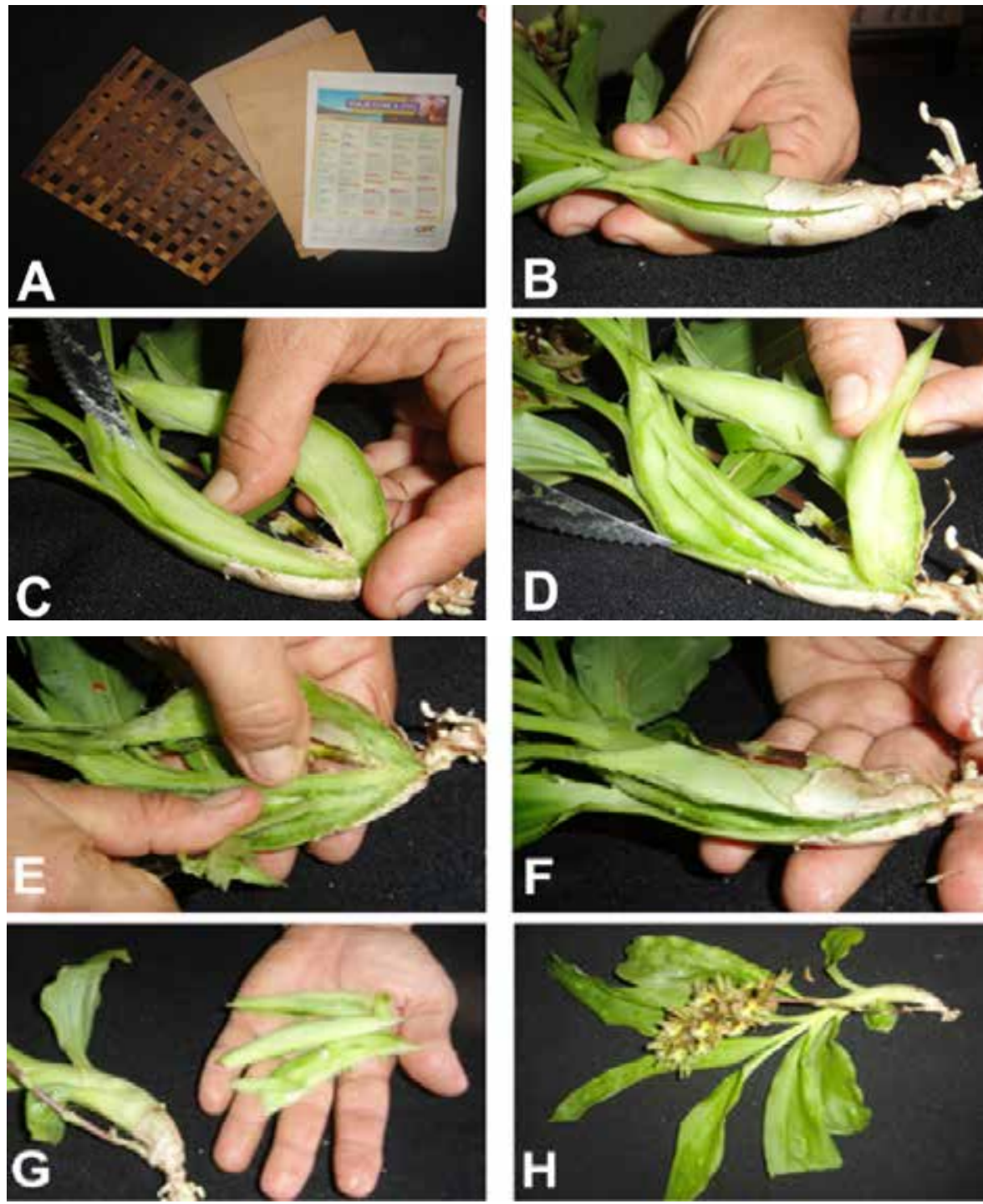

Figura 1. A - Material básico de herborização: prensa de madeira, recortes de papelão e folhas de jornal; B a F - Passo-a-passo do corte do pseudobulbo de uma amostra de Catasetum arietinum e retirada da mucilagem interna; $\mathrm{G}$ - Material mucilaginoso retirado do pseudobulbo; $\mathrm{H}$ - Amostra com as partes do pseudobulbo unidas, pronta para prensagem. Fotos: Stacy Anna P. Benelli.

O material utilizado no processo é o tradicional de herborização, sendo: prensa de madeira, jornais e cortes de papelão (Figura $1 \mathrm{~A}$ ). $\mathrm{O}$ processo de secagem foi realizado sob o calor do sol, mas pode ser em estufa elétrica, caso esteja disponível 
ao herborizador. O corte das amostras foi realizado com uma faca de ponta aguda e lâmina estreita, curta e afiada (de preferência, facas serrilhadas tipo Ginsu ${ }^{\circledR}$ ). Quando necessário, a operação foi complementada, utilizando-se uma colher para retirar o máximo de mucilagem possível.

Preparação da amostra para exsicar - O processo descrito caracteriza-se pela simplicidade e facilidade de reprodução e consiste de passos complementares aos já conhecidos para a herborização de amostras botânicas (FIDALGO; BONONI, 1989), sendo eles:

1. Com uma faca de lâmina estreita, foi realizado um corte no sentido longitudinal do pseudobulbo, acompanhando a linha central das brácteas foliares (laterais dos pseudobulbos), sem seccioná-lo por completo (Figura 1 B);

2. Com o pseudobulbo aberto em duas metades procedeu-se com cortes nas bordas interiores de cada banda (Figura $1 \mathrm{C}$ ), visando retirar o conteúdo todo de uma só vez, em bloco (Figura $1 \mathrm{D}$ );

3. Cuidadosamente, foram retirados todos os restos da mucilagem que se encontra aderida às paredes internas (Figura $1 \mathrm{E}$ ) e realizada a junção das duas metades (Figura $1 \mathrm{~F}$ ), formando novamente o pseudobulbo, desta vez totalmente oco.

A grande quantidade de mucilagem que foi retirada de apenas um pseudobulbo de Catasetum é mostrada na Figura $1 \mathrm{G}$. Esse material, muito úmido devido à grande quantidade de água contido nele, seria um grande transtorno no processo de secagem da amostra caso não fosse retirado.

A amostra, após a montagem final, foi preparada para a montagem de uma exsicata perfeita, de modo que não se perceba, após a secagem, que o pseudobulbo foi seccionado e, o mais importante, de forma que a amostra demonstre sua morfologia, sem distorções (Figura $1 \mathrm{H}$ ). A secagem da amostra após esse preparo se dá mais homogeneamente e em poucos dias, em média, 1/3 do que seria no processo padrão.

Após a total secagem da amostra, ela foi colada (Figura 2 A) sobre a cartolina para ser depositada no herbário. O procedimento de fixação da amostra na cartolina (colada, costurada ou fixada com fitilho), bem como as dimensões desta, variam de uma instituição para outra. Uma ficha com todos os dados de coleta e as demais informações relativas à amostra foi preparada e anexada à cartolina (Figura 2 B). Também nesse caso, a posição onde essa etiqueta deve ser colada varia dependendo 
da instituição, sendo que a maioria adota a sua colocação no canto inferior direito. Essa ficha foi fixada utilizando um fio de cola na borda direita (Figura $2 \mathrm{C}$ ). Dessa forma, além de possibilitar a consulta de parte da amostra que fique coberta pela etiqueta, ela ainda poderá ser retirada no herbário para o qual for encaminhada, caso o procedimento deste seja diferente ou adotem etiqueta própria. No caso de ser material a ser encaminhado para herbários distantes, é recomendado que se prepare uma capa com papel de seda para proteger a amostra (Figura 2 D).
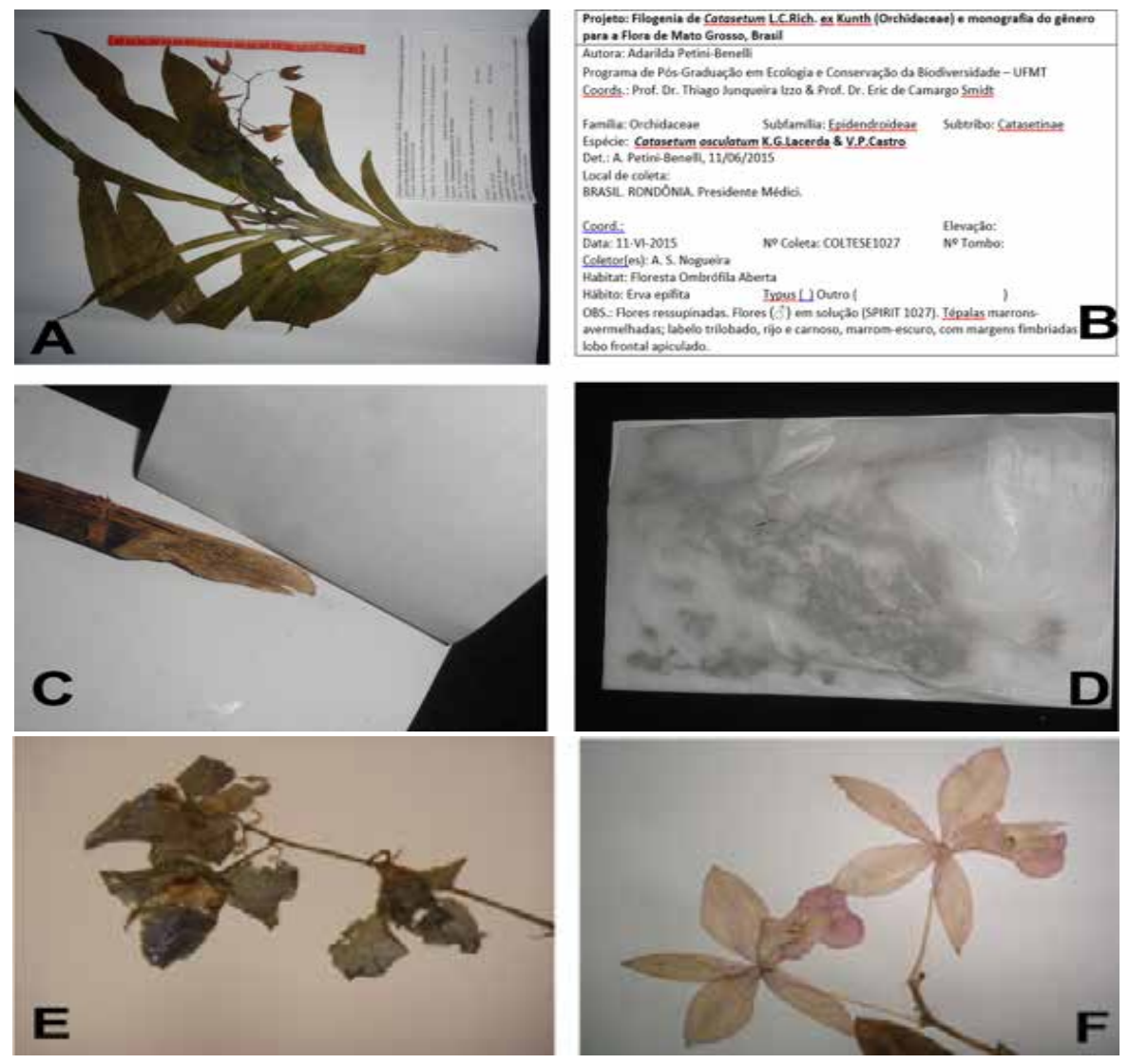

Figura 2. A - Amostra de Catasetum confusum após secagem, sendo colada à cartolina; B - Ficha de Identificação que será colada à cartolina junto com a respectiva amostra, contendo os dados de coleta, determinação e florística; C - Forma indicada para colagem da Ficha de Identificação na cartolina; D - Capa preparada com papel de seda para proteção da amostra após sua preparação; E Amostra de Aganisia cyanea preparada em maio de 2006, conforme as técnicas apresentadas neste trabalho, mostrando parte de sua coloração original preservada; F - Amostra de Cattleya nobilior, preparada em abril de 2007 e que também manteve muito de sua coloração original.

Fonte: Acervo do autor (2015) 


\section{RESULTADOS E DISCUSSÃO}

Essa técnica foi utilizada na herborização de amostras de dezenas de espécies, por exemplo, dos gêneros Aganisia Lindl., Aspasia Lindl., Catasetum Rich. ex Kunth, Cattleya Lindl., Cycnoches Lindl., Cyrtopodium R.Br., Galeandra Lindl., Mormodes Lindl., Xylobium Lindl. e Zygosepalum Rchb.f. (Quadro 1). Em todos os espécimes em que a mucilagem foi retirada por esse processo, registrouse uma redução de até $70 \%$ no tempo de secagem. Em Aganisia cyanea (Lindl.) Rchb.f. (Figura 2 E), Catasetum multifidum F.E.L.Miranda (Figura 2 F) e Cattleya nobilior Rchb.f., por exemplo, houve a preservação da coloração das flores, o que é um resultado relevante.

Quadro 1. Relação de material testemunha preparado com a técnica descrita nesse trabalho e depositado em herbários brasileiros.

(Continua)

\begin{tabular}{|c|c|}
\hline Espécie & Coletor $/ \mathbf{n}^{0}$ coletor (Acervo/ $\mathbf{n}^{0}$ tombo) \\
\hline Aganisia cyanea (Lindl.) Rchb.f. & $\begin{array}{l}\text { A. Petini-Benelli } 25 \text { (UFMT 36328) } \\
\text { A. Petini-Benelli } 26 \text { (UFMT 36339) } \\
\text { A. Petini-Benelli } 27 \text { (UFMT 36329) }\end{array}$ \\
\hline $\begin{array}{l}\text { Alatiglossum culuenense Docha-Neto \& } \\
\text { Benelli }\end{array}$ & $\begin{array}{l}\text { A. Petini-Benelli 36-PCHP2 (Holótipo: UFMT 36666) } \\
\text { A. Petini-Benelli 37-PCHP2 (Parátipo: UFMT 36667) }\end{array}$ \\
\hline Aspasia variegata Lindl. & A. Petini-Benelli APB931 (RB 597045) \\
\hline $\begin{array}{l}\text { Catasetum } \times \text { altaflorestense Benelli \& } \\
\text { Grade }\end{array}$ & $\begin{array}{l}\text { A. Grade ADA1253 (HERBAM - Falta) } \\
\text { A. Petini-Benelli ADA028 (UFMT 40845) } \\
\text { A. Petini-Benelli s.n. (Parátipo: UFMT 38635) } \\
\text { A. Petini-Benelli; A. Grade s.n. (Holótipo: UFMT 38636) }\end{array}$ \\
\hline Catasetum $\times$ apolloi Benelli \& Grade & $\begin{array}{l}\text { A. Grade et al. 59-SNC (Holótipo: UFMT 37269) } \\
\text { A. Grade et al. 60-SNC (Holótipo: UFMT 37270) }\end{array}$ \\
\hline $\begin{array}{l}\text { Catasetum arietinum F.E.L.Miranda \& } \\
\text { K.G.Lacerda }\end{array}$ & $\begin{array}{l}\text { P.M. Pinheiro ADA1260 (UFMT 41446) } \\
\text { T. César ADA1277 (RB 611358) }\end{array}$ \\
\hline Catasetum barbatum (Lindl.) Lindl. & $\begin{array}{l}\text { A. Petini-Benelli; A.S. Albuquerque ADA1162 (RB 597047; UFMT 41226) } \\
\text { S.A.Queiroz ADA875 (UFMT 41228) }\end{array}$ \\
\hline Catasetum blackii Pabst & A.S. Albuquerque ADA309 (UFMT 42504) \\
\hline $\begin{array}{l}\text { Catasetum complanatum F.E.L.Miranda \& } \\
\text { K.G.Lacerda }\end{array}$ & A. Petini-Benelli; G. Lima-Alves Jr. ADA36 (UFMT 40837) \\
\hline Catasetum denticulatum F.E.L.Miranda & $\begin{array}{l}\text { A. Petini-Benelli ADA558 (RB 594053) } \\
\text { A. Petini-Benelli; A.S. Nogueira ADA542 (IAN 192013; UPCB) } \\
\text { A.S. Nogueira ADA543 (HBR 54449) } \\
\text { S.C. Freitas ADA568 (UFMT 41234) }\end{array}$ \\
\hline Catasetum discolor Lindl. & $\begin{array}{l}\text { A. Petini-Benelli ADA236 (UFMT 41383) } \\
\text { A. Petini-Benelli ADA398 (UFMT 41224) }\end{array}$ \\
\hline
\end{tabular}


(Continua)

\begin{tabular}{|c|c|}
\hline Espécie & Coletor $/ \mathbf{n}^{0}$ coletor (Acervo/ $\mathbf{n}^{0}$ tombo) \\
\hline Catasetum discolor Lindl. & A. Grade; H.S. Lima ADA40 (UFMT 40835) \\
\hline Catasetum $\times$ faustoi Bicalho & S.C. Freitas ADA220 (UFMT 41384) \\
\hline $\begin{array}{l}\text { Catasetum fimbriatum (C.Morren) Lindl. } \\
\text { \& Paxton }\end{array}$ & $\begin{array}{l}\text { A. Petini-Benelli ADA20 (RB 597049) } \\
\text { A. Petini-Benelli ADA280 (UFMT 41409) } \\
\text { A. Petini-Benelli; E. Sanches ADA560 (HBR 54450; RB 594043; UPCB) } \\
\text { A. Petini-Benelli; N. Monteiro ADA353 (UPCB) } \\
\text { A. Petini-Benelli; N. Monteiro ADA369 (UPCB) } \\
\text { C.R. Martins ADA025 (UFMT 42503) } \\
\text { C.R. Martins ADA232 (UPCB - Falta) } \\
\text { N. Monteiro ADA349 (IAN) }\end{array}$ \\
\hline Catasetum fimbriatum var. fissum Rchb.f. & C.M. Régent ADA02 (UFMT 40843) \\
\hline $\begin{array}{l}\text { Catasetum fimbriatum Rchb.f. var. } \\
\text { morrenianum Mansf. }\end{array}$ & A. Petini-Benelli ADA01 (UFMT 40842) \\
\hline Catasetum $\times$ freitasii Benelli & J. Fernández G. et al. ADA1230a (Holótipo: UFMT 41208) \\
\hline Catasetum fuchsii Dodson \& R.Vásquez & J.B. Pinho ADA1173 (UFMT 41206) \\
\hline Catasetum galeritum Rchb.f. & $\begin{array}{l}\text { A. Petini-Benelli ADA1256 (UFMT 41401) } \\
\text { R.P. Andrade ADA33 (UFMT 40841) } \\
\text { R.P. Andrade ADA1191 (UFMT 41452) }\end{array}$ \\
\hline Catasetum gardneri Schltr. & $\begin{array}{l}\text { R.S. Lopes ADA06 (UFMT 41387) } \\
\text { T.E.C. Meneguzzo ADA1410 (UFMT 42510) }\end{array}$ \\
\hline Catasetum gladiatorium K.G.Lacerda & $\begin{array}{l}\text { A. Petini-Benelli ADA366 (RB 594047) } \\
\text { A. Petini-Benelli; N. Monteiro ADA240 (HBR 54451; IAN 192011) } \\
\text { A. Petini-Benelli; N. Monteiro ADA253 (UPCB) } \\
\text { A. Petini-Benelli; N. Monteiro ADA370 (IAN; UPCB) } \\
\text { A. Petini-Benelli; N. Monteiro ADA374 (UPCB) }\end{array}$ \\
\hline Catasetum gnomus L.Linden \& Rchb.f. & A.S. Nogueira ADA547 (RB 597046) \\
\hline Catasetum bookeri Lindl. & $\begin{array}{l}\text { M.L.N. Duba ADA1168 (UPCB) } \\
\text { M.L.N. Duba ADA1176 (UFMT 41227) }\end{array}$ \\
\hline Catasetum juruenense Hoehne & $\begin{array}{l}\text { A. Petini-Benelli ADA12 (UFMT 41189) } \\
\text { A. Petini-Benelli ADA278 (RB 597042) } \\
\text { A. Petini-Benelli ADA290 (UPCB) }\end{array}$ \\
\hline Catasetum lanciferum Lindl. & N.M. Monteiro ADA249 (UFMT 41187) \\
\hline Catasetum luridum (Link \& Otto) Lindl. & W.M. Alves ADA1139 (UFMT 41223) \\
\hline Catasetum macrocarpum Rich. ex Kunth & $\begin{array}{l}\text { A. Petini-Benelli ADA35 (RB 612178; UFMT 40839) } \\
\text { A. Petini-Benelli ADA247 (UFMT 41237) } \\
\text { A. Petini-Benelli ADA408 (IAN) } \\
\text { A. Petini-Benelli; N. Monteiro ADA242 (UPCB) } \\
\text { A. Petini-Benelli; N. Monteiro ADA250 (RB 597033) } \\
\text { K. Rodrigues ADA1345 (UFMT 42506) } \\
\text { R.P. Andrade ADA38 (HERBAM; UPCB) } \\
\text { R.P. Andrade ADA1145 (RB 597034) } \\
\text { R.P. Andrade ADA1183 (UFMT 41395) }\end{array}$ \\
\hline Catasetum mattosianum Bicalho & $\begin{array}{l}\text { A. Petini-Benelli ADA195 (UFMT 41408) } \\
\text { W.M. Alves ADA204 (UFMT 42494) }\end{array}$ \\
\hline
\end{tabular}


(Continua)

\begin{tabular}{|c|c|}
\hline Espécie & Coletor $/ \mathbf{n}^{0}$ coletor (Acervo/n $\mathbf{n}^{0}$ tombo) \\
\hline $\begin{array}{l}\text { Catasetum osculatum K.G.Lacerda \& } \\
\text { V.P.Castro }\end{array}$ & $\begin{array}{l}\text { A. Petini-Benelli 22-JBH (UFMT 36448) } \\
\text { A. Petini-Benelli 67-SSV (UFMT 38240) } \\
\text { A. Petini-Benelli ADA05 (UFMT 40846) } \\
\text { A. Petini-Benelli ADA34 (UFMT 40840) } \\
\text { A. Petini-Benelli ADA269 (RB 597039) } \\
\text { A. Petini-Benelli ADA270 (IAN 192992; UFMT) } \\
\text { A. Petini-Benelli ADA273 (HERBAM; IAN 192993) } \\
\text { A. Petini-Benelli ADA578 (HERBAM) } \\
\text { A. Petini-Benelli ADA581 (HBR 54452) } \\
\text { A. Petini-Benelli ADA1137 (UPCB) } \\
\text { A. Petini-Benelli ADA1140 (RB 597036) } \\
\text { A. Petini-Benelli ADA1147 (RB 597035) } \\
\text { A. Petini-Benelli ADA1165 (RB 597048) } \\
\text { A. Petini-Benelli ADA1166 (UPCB) } \\
\text { A. Petini-Benelli ADA1189 (IAN 192996) } \\
\text { A. Petini-Benelli ADA1231 (HBR 54453) } \\
\text { A. Petini-Benelli ADA1232 (HBR 54454; IAN; UPCB) } \\
\text { A. Petini-Benelli APB834 (UFMT 40849) } \\
\text { A. Petini-Benelli et al. ADA221 (UPCB) } \\
\text { A. Petini-Benelli et al. ADA378 (UFMT 41432) } \\
\text { A. Petini-Benelli; G. Lima-Alves Jr 4-LTRO (UFMT 40834) } \\
\text { A. Petini-Benelli et al. ADA341 (RB 612180) } \\
\text { A.S. Nogueira ADA552 (IAN 192994) } \\
\text { A.S. Nogueira APB956 (RB 612176) } \\
\text { J. Santana ADA1161 (UPCB) } \\
\text { J. Santana ADA1164 (RB 597050) } \\
\text { M. Garcia ADA1254 (UFMT 41449) } \\
\text { M. Garcia APB933 (IAN 192990) } \\
\text { M. Garcia APB935 (RB 612177) } \\
\text { M. Garcia APB975 (IAN 192991) } \\
\text { M.R. Garcia ADA1342 (UFMT 42501) } \\
\text { R.P. Andrade ADA1234 (IAN; UFMT 41428) } \\
\text { R.P. Andrade ADA1252 (UFMT 41429) } \\
\text { R. Soares ADA1158 (IAN 192995) } \\
\text { S.C. Freitas ADA192 (UPCB) } \\
\text { S.C. Freitas ADA400 (RB 597030) } \\
\text { P. }\end{array}$ \\
\hline Catasetum pileatum Rchb.f. & A. Petini-Benelli ADA216 (UPCB) \\
\hline Catasetum planiceps Lindl. & A. Schmidt; S.C. Freitas ADA029 (UFMT 41186) \\
\hline Catasetum purum Nees \& Sinning & A. Petini-Benelli ADA27 (UPCB) \\
\hline Catasetum rooseveltianum Hoehne & $\begin{array}{l}\text { A. Petini-Benelli 23-JBH (UFMT 36447) } \\
\text { A. Petini-Benelli ADA1163 (RB 597051; UFMT 41240) } \\
\text { A. Petini-Benelli ADA1235 (RB 597032) } \\
\text { A. Petini-Benelli et al. ADA396 (UFMT 41405) } \\
\text { R.F. Nunes APB961 (UFMT) } \\
\text { R.P. Andrade ADA165 (UFMT 40859) } \\
\text { R.P. Andrade ADA166 (UFMT 40860) } \\
\text { R.P. Andrade ADA167 (UFMT 41394) }\end{array}$ \\
\hline Catasetum saccatum (Lindl.) & A. Petini-Benelli ADA188 (RB 597040) \\
\hline $\begin{array}{l}\text { Catasetum schmidtianum } \text { F.E.L.Miranda \& } \\
\text { K.G.Lacerda }\end{array}$ & $\begin{array}{l}\text { A. Petini-Benelli; J.H. Piva s.n. (RB 597041) } \\
\text { A. Petini-Benelli; J.H. Piva ADA04 (UFMT 40848) } \\
\text { A. Petini-Benelli; J.H. Piva ADA231 (RB 612175) }\end{array}$ \\
\hline
\end{tabular}


(Continua)

\begin{tabular}{|c|c|}
\hline Espécie & Coletor $/ \mathbf{n}^{\mathbf{o}}$ coletor (Acervo/ $\mathbf{n}^{\mathbf{0}}$ tombo) \\
\hline Catasetum semicirculatum F.E.L.Miranda & S.C. Freitas ADA31 (UFMT 40844) \\
\hline Catasetum socco (Vell.) Hoehne & $\begin{array}{l}\text { A. Petini-Benelli ADA1249 (UFMT 42509) } \\
\text { A. Petini-Benelli; A.S. Medeiros ADA1244 (RB 610537; UFMT 42502) } \\
\text { A. Petini-Benelli; A.S. Medeiros ADA1245 (UFMT 42500) } \\
\text { F. Mariotto ADA1146 (UPCB) }\end{array}$ \\
\hline Catasetum $\times$ tapiriceps Rchb.f. & R.P. Andrade ADA299 (UFMT 42497) \\
\hline $\begin{array}{l}\text { Catasetum } \times \text { valdisonianum } \\
\text { U.L.C.Ferreira }\end{array}$ & S.C. Freitas ADA308 (UFMT 41442) \\
\hline Catasetum vinaceum Hoehne & $\begin{array}{l}\text { A. Petini-Benelli ADA582 (UFMT 41232) } \\
\text { A. Petini-Benelli ADA1141 (UFMT 41229) } \\
\text { A. Petini-Benelli; N. Monteiro ADA303 (RB 597029) }\end{array}$ \\
\hline Catasetum sp. & A. Grade; A. Petini-Benelli (UFMT 30849) \\
\hline Catasetum sp. & A. Grade; H.S. Lima s/no (UFMT 37346) \\
\hline Catasetum sp. & A. Petini-Benelli ADA03 (UFMT 40847) \\
\hline Catasetum sp. & A. Petini-Benelli ADA03A (UFMT 40833) \\
\hline Catasetum sp. & A. Petini-Benelli ADA238 (UFMT 41231) \\
\hline Catasetum sp. & P.R. Paêlo ADA562a (UFMT 41211) \\
\hline Cattleya $\times$ mesquitae L.C.Menezes & A. Petini-Benelli; J. Vilela-Neto APB895 (UFMT 41196) \\
\hline Cattleya nobilior Rchb.f. & $\begin{array}{l}\text { A. Petini-Benelli APB889 (UFMT 40858) } \\
\text { A.Petini-Benelli; P.R. Paelo, 10-PCHP (UFMT 36358) } \\
\text { A.Petini-Benelli; P.R. Paelo, 11-PCHP (UFMT 36360) } \\
\end{array}$ \\
\hline Cycnoches haagii Barb.Rodr. & $\begin{array}{l}\text { A. Petini-Benelli ADA1175 (UFMT 41407) } \\
\text { A. Petini-Benelli ADA1184 (UFMT 41406) }\end{array}$ \\
\hline $\begin{array}{l}\text { Cycnoches manoelae V.P.Castro \& } \\
\text { Campacci }\end{array}$ & A. Petini-Benelli ADA377 (UFMT 41222) \\
\hline Cycnoches pentadactylon Lindl. & $\begin{array}{l}\text { A.S. Nogueira ADA537 (UFMT 41230) } \\
\text { A.S. Nogueira ADA538 (UFMT 41440) }\end{array}$ \\
\hline Cyrtopodium paludicola Hoehne & A. Petini-Benelli 89 (UFMT 38229) \\
\hline Cyrtopodium saintlegerianum Rchb.f. & $\begin{array}{l}\text { A. Petini-Benelli; R.P. Benelli ADA1294 (HERBAM; IAN 192997; RB } \\
612101 ; \text { UFMT) }\end{array}$ \\
\hline Encyclia argentinensis (Speg.) Hoehne & $\begin{array}{l}\text { A. Petini-Benelli 52-PRF (UFMT 37159) } \\
\text { A. Petini-Benelli 53-PRF (UFMT 37158) } \\
\text { A. Petini-Benelli APB926 (RB 594044) } \\
\text { A. Petini-Benelli APB927 (RB 594052) } \\
\end{array}$ \\
\hline $\begin{array}{l}\text { Encyclia clovesiana L.C.Menezes \& } \\
\text { V.P.Castro }\end{array}$ & A. Petini-Benelli APB928 (RB 594052) \\
\hline Encyclia saltensis Hoehne & J. Pañozzo 405 (UFMT 27779) \\
\hline Galeandra blanchetii E.S.Rand & $\begin{array}{l}\text { A. Petini-Benelli ADA271 (HERBAM; UPCB) } \\
\text { A. Petini-Benelli ADA274 (RB 594041) } \\
\text { A. Petini-Benelli ADA379 (UPCB) } \\
\text { A. Petini-Benelli ADA405 (HERBAM) } \\
\text { A. Petini-Benelli ADA406 (IAN 192012) } \\
\text { A. Petini-Benelli ADA409 (RB 594042) } \\
\text { A. Petini-Benelli ADA411 (UFMT 41193) } \\
\text { A. Petini-Benelli ADA559 (HBR 54455) } \\
\text { A. Petini-Benelli ADA561 (RB 612181) } \\
\text { A. Petini-Benelli ADA564 (IAN 192015) }\end{array}$ \\
\hline
\end{tabular}




\begin{tabular}{|c|c|}
\hline Espécie & Coletor $/ \mathbf{n}^{0}$ coletor (Acervo/ $/ \mathbf{n}^{0}$ tombo) \\
\hline Galeandra blanchetii E.S.Rand & $\begin{array}{l}\text { A. Petini-Benelli ADA565 (HBR 54456; UPCB) } \\
\text { R. Soares ADA1240 (UFMT 41450) }\end{array}$ \\
\hline Galeandra stangeana Rchb.f. & $\begin{array}{l}\text { A. Petini-Benelli ADA1152 (RB 597052) } \\
\text { A. Petini-Benelli ADA1153 (RB 597053) } \\
\text { A. Petini-Benelli ADA1233 (HERBAM) } \\
\text { A. Petini-Benelli APB941 (UPCB) } \\
\text { R. Soares ADA1148 (UFMT 41241) } \\
\text { R. Soares ADA1149 (UFMT 41220) } \\
\text { R. Soares ADA1151 (UFMT 41438) } \\
\text { R. Soares ADA1154 (IAN 192998; UFMT 41441) } \\
\text { R. Soares APB937 (UFMT 41217) }\end{array}$ \\
\hline $\begin{array}{l}\text { Galeottia ciliata (C.Morel) Dressler \& } \\
\text { Christenson }\end{array}$ & A. Petini-Benelli 64 (UFMT 38230) \\
\hline Laelia gloriosa (Rchb.f.) L.O.Williams & $\begin{array}{l}\text { C.R.A. Soares et al. 3608a (HERBAM 5482) } \\
\text { C.R.A. Soares et al. 3608b (HERBAM 5623) } \\
\text { C.R.A. Soares et al. 3608c (HERBAM 5624) } \\
\end{array}$ \\
\hline $\begin{array}{l}\text { Lycaste rossiana var. mattogrossensis Barb. } \\
\text { Rodr. }\end{array}$ & A. Petini-Benelli s.n. (UFMT 38450) \\
\hline Prosthechea vespa (Vell.) W.E.Higgins & $\begin{array}{l}\text { A. Petini-Benelli } 49 \text { (UFMT 36858) } \\
\text { A. Petini-Benelli } 51 \text { (UFMT 36859) } \\
\text { A. Petini-Benelli; S.C. Freitas APB923 (RB 594050; UFMT 41190) }\end{array}$ \\
\hline Xylobium foveatum (Lindl.) Nichols. & $\begin{array}{l}\text { C.R.A. Soares et al. 3701a (HERBAM 5528) } \\
\text { C.R.A. Soares et al. 3701b (HERBAM 5631) } \\
\text { C.R.A. Soares et al. 3701c (HERBAM 5632) }\end{array}$ \\
\hline Zygosepalum labiosum (Rich.) Garay & C.R.A. Soares et al. 3257a (HERBAM 5191) \\
\hline
\end{tabular}

Fonte: Nomenclatura adotada por INPI - International Names Plant Index (http://www.ipni.org).

Em alguns experimentos, obtiveram-se resultados rápidos com a exposição direta ao sol, por pelo menos $5 \mathrm{~h} /$ dia, da prensa montada com as amostras. Até o momento, mais de 200 exsicatas já foram preparadas (Quadro 1), a maioria de Catasetinae, sem a utilização de eletricidade, obtendo-se vouchers de excelente aspecto (Figura 2).

Em Fidalgo e Bononi (1989) há a orientação para seccionar os caules espessos, referindo-se a Araceae. Tal técnica, ao ser utilizada em pseudobulbos de Catasetum, mostrou-se insatisfatória, pois tais órgãos se retorceram, inclusive dando a ilusão de que tinham uma morfologia diferente da que é natural da espécie, além do processo de secagem continuar demorado.

A técnica de retirada de mucilagem dos pseudobulbos pode ser aplicada a qualquer amostra que possua pseudobulbos engrossados, bulbos suculentos, cáu- 
dex ou cladódios (como os de Cactaceae), auxiliando aos pesquisadores e otimizando o processo de herborização de amostras em campo, laboratório ou herbário.

\section{CONSIDERAÇÕES FINAIS}

Sabendo que deixar o material secando por muito tempo na estufa desidrata excessivamente as folhas e flores, deixando o material quebradiço, buscou-se uma forma de acelerar o processo de secagem de amostras de Catasetinae. Concluiu-se que retirando a parte interna do pseudobulbo haveria grande redução no tempo de secagem e os danos relatados acima não aconteceriam. Após algumas tentativas e erros, conseguiu-se os resultados esperados de forma simples e rápida.

A facilidade do processo de preparação das amostras de Catasetum e o excelente resultado obtido foram o maior estímulo para a descrição dessa técnica, além das várias solicitações para sua transmissão em treinamentos e cursos de Técnicas de Coleta e Herborização, nos Herbários UFMT e HERBAM. Outro aspecto positivo da aplicação dessa técnica é que possibilita a redução no consumo de energia, tanto na estufa de lâmpadas incandescentes, quanto na estufa elétrica.

\section{AGRADECIMENTOS}

Agradecimento especial à Stacy Anna Petini Benelli, pelo suporte com as fotografias. A Cristiano Pedroso de Moraes, Thiago Erir Cadete Meneguzzo e aos avaliadores anônimos pelas críticas oportunas. A Thiago Semedo pela preparação das figuras. Aos curadores dos herbários pelo suporte no acesso às coleções. À CAPES - Coordenação de Aperfeiçoamento de Pessoal de Nível Superior pelo aporte financeiro.

\section{REFERÊNCIAS}

BENZING, D. H. The vegetative basis of vascular epiphytism. Selbyana v. 9, p. 23-43, 1986. 
BENZING, D. H. Vascular epiphytism: taxonomic participation and adaptative diversity. Annals of the Missouri Botanical Garden, v. 74, n. 2, p. 183-204, 1987.

DRESSLER, R. L. The Orchids: natural history and classification. Harvard University Press, London, 1990.

FIDALGO, O.; BONONI, V. L. R. (Coord.) Técnicas de coleta, preservação e herborização de material botânico. São Paulo: Instituto de Botânica de São Paulo, 1989. 62 p. (Série Documentos),

HOEHNE, F. C. Orchidaceae. In: HOEHNE, F. C. Flora Brasilica, v. 12, n. 6, p. 1-254, 1942.

NG, C. K. Y.; HEW, C. S. Orchid pseudobulbs - 'false' bulbs with a genuine importance in orchid growth and survival! Scientia Horticulturae, v. 83, p. 165-172, 2000.

OLIVEIRA, V. C.; SAJO, M. G. Morfo-Anatomia caulinar de nove espécies de Orchidaceae. Acta botanica brasílica, v. 15, n. 2, p. 177-188, 2001.

PEDROSO-DE-MORAES, C.; SOUZA-LEAL, T.; BRESCANSIN, R. L.; PETINI-BENELLI, A.; SAJO, M. G. Radicular anatomy of twelve representatives of the Catasetinae subtribe (Orchidaceae: Cymbidieae). Anais da Academia Brasileira de Ciências, v. 84, n. 2 , p. 455-467, 2012.

ROMERO, G. A.; CARNEVALI, G. Description [Catasetum], p. 13-14. In: PRIDGEON, A. M.; CRIBB, P. J.; CHASE, M. W.; RASMUSSEN, F. N. (Ed.). Genera Orchidacearum. Oxford University Press, 2009. 586 p. vol. 5 Epidendroideae (Part two).

ROTTA, E.; BELTRAMI, L. C. C.; ZONTA, M. Manual de prática de coleta e herborização de material botânico. Colombo: Embrapa Florestas, 2008.

STERN, W. L.; JUDD, W. S. Comparative anatomy and systematics of Catasetinae (Orchidaceae). Botanical Journal of the Linnean Society v. 136, p. 153-178, 2001.

STERN, W. L.; CARLSWARD, B. S. Comparative vegetative anatomy and systematics of the Oncidiinae (Maxillarieae, Orchidaceae). Botanical Journal of the Linnean 
Society v. 152, p. 91-107, 2006.

Recebido em: 30 de junbo de 2015

Revisado em: 14 de outubro de 2015

Aceito em: 22 de dezembro de 2015 